\title{
Aktuelle Rechtsprechung in der Radiologie - Kostenerstattung für PET-CT/MRT-Untersuchungen und keine CT-Abrechnungsgenehmigung für Doppelfachärzte
}

\section{Kostenerstattung für PET-CT/MRT-Untersuchung zur Tumordiagnostik gebilligt}

\section{Einführung}

Auch nicht anerkannte Behandlungsmethoden können unter bestimmten Voraussetzungen der Leistungspflicht der gesetzlichen Krankenversicherung (GKV) unterfallen.

Maßgeblich für eine Kostenübernahme durch die GKV ist insbesondere $\S 13$ Fünftes Buch Sozialgesetzbuch (SGB V). Die Kostenerstattung einer anerkannten als auch einer nicht anerkannten Behandlungsmethode ist grundsätzlich möglich, sofern die Antragstellung der Kostenübernahme vor Inanspruchnahme der Leistung erfolgte und die Krankenkasse die Kostenübernahme zugesagt oder nicht fristgemäß reagiert hat, §13 Abs. 2 und Abs. 3a SGB V. Die Kosten für selbst beschaffte Leistungen sind von der Krankenkasse zu erstatten, soweit die Leistung notwendig war, wenn die Krankenkasse eine unaufschiebbare Leistung nicht rechtzeitig erbringen konnte oder sie eine Leistung zu Unrecht abgelehnt hat und dadurch dem Versicherten für die selbstbeschaffte Leistung Kosten entstanden sind, § 13 Abs. 3 SGB V.

Damit die Kosten einer nicht anerkannten Behandlungsmethode außerhalb des Leistungskataloges durch die GKV übernommen werden können, müssen nach der Rechtsprechung des Bundesverfassungsgerichts (BVerfG) besondere Voraussetzungen erfüllt sein, welche anhand der folgenden Entscheidung näher dargestellt werden.

\section{Entscheidung des Sozialgerichts Leipzig}

Das Sozialgericht (SG) Leipzig hat mit Gerichtsbescheid vom 22.04.2020, Az.: S 8 KR 1743/19 entschieden, dass ein Patient die Erstattung der Kosten in Höhe von 933,49€ für eine Positronen-EmissionsTomographie-Computertomographie/Magnetresonanztomographie (PET-CT/MRT) ver- langen kann. Zwar liegt für eine PET-CT/ MRT-Untersuchung bei Hodentumor und mehreren abgebrochenen Chemotherapien keine positive Bewertung oder Empfehlung des Gemeinsamen Bundesausschusses (G-BA) vor. Eine Kostenübernahme ist gleichwohl trotzdem möglich, wenn die durch das BVerfG aufgestellten Voraussetzungen vorliegen. Ansprüche, die diagnostische Maßnahmen zum Gegenstand haben, sind als Krankenbehandlung davon ebenfalls erfasst. Die erforderliche spürbare positive Einwirkung auf den Krankheitsverlauf kann daher auch darin liegen, lebensbedrohliche Risiken von Therapieoptionen überhaupt erst zu eruieren.

\section{a. Sachverhalt}

Der Patient verlangte von seiner gesetzlichen Krankenkasse die Kostenübernahme für eine PET-CT/MRT. Er litt an einem Hodentumor Stadium III. Aufgrund dessen unterzog er sich mehreren Chemotherapien. Er beantragte die Kostenübernahme für eine PET-CT/MRT unter Vorlage eines Kostenvoranschlages des Universitätsklinikums. Laut Anmeldung solle geprüft werden, ob noch vitales Tumorgewebe bestehe.

Die Krankenkasse lehnte eine Kostenübernahme ab. Es handele sich um keine von den gesetzlichen Krankenkassen zu übernehmende Leistung, weil dafür der diagnostische bzw. therapeutische Nutzen nachgewiesen sein müsse. Der G-BA habe den Nutzen einer PET/PET-CT aber nur für bestimmte medizinische Indikationen festgestellt, für die sie ausnahmsweise die Kosten tragen könne - eine solche läge bei dem Patienten jedoch (zutreffend) nicht vor.

Hiergegen legte der Patient Widerspruch ein. Nach Stellungnahme der behandelnden Fachärztin für Urologie befinde sich ihr Patient wegen seines schwerwiegenden Krebsleidens in ihrer ambulanten urologischen Behandlung. Aufgrund erheblicher Nebenwirkungen, vor allem Panzytopenie, sei er einem deutlich erhöhten Infektions-

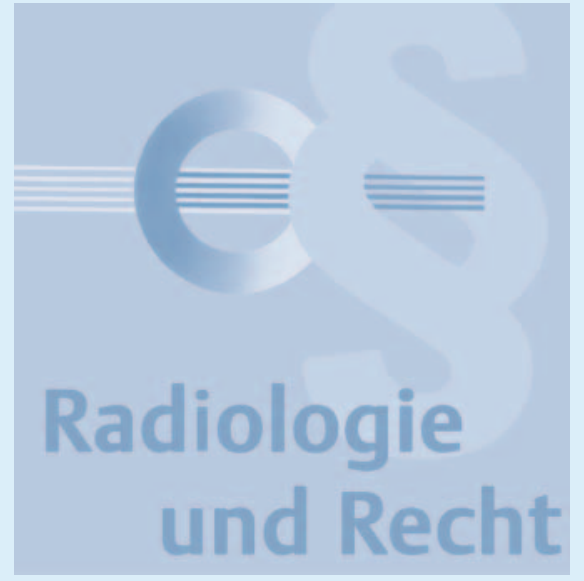

risiko ausgesetzt, weshalb die Chemotherapie mehrfach habe unterbrochen werden müssen. Nach Abschluss von drei, aufgrund von Nebenwirkungen nur unvollständig durchführbaren Zyklen Chemotherapie und nach Besprechung des Falls im Tumorboard habe sich das Krankenhaus entschieden, die Durchführung einer PSMA PET-CT zu empfehlen. Nach deren Durchführung könne der Patient, bei bestehendem Nachweis von vitalen Tumorarealen, an ein Zentrum mit der Möglichkeit der Knochenmarkasservierung, beispielsweise an das Universitätsklinikum, zur Einleitung einer Chemotherapie zugewiesen werden; denn im Hinblick auf die Unterscheidung von nekrotischem und vitalem Tumorgewebe reiche eine herkömmliche Bildgebung nicht aus. Bei geringstem Zweifel und aufgrund der drohenden schwerwiegenden Komplikationen könne eine Chemotherapie nicht „leichtfertig“ durchgeführt werden, die daraus folgenden möglichen Nebenwirkungen könnten für den Patienten lebensbedrohlich seien.

Gegen den ablehnenden Widerspruchsbescheid der Krankenkasse hat der Patient schließlich Klage zum Sozialgericht Leipzig erhoben.

\section{b. Begründung}

Das grundsätzliche Sachleistungsprinzip der Krankenkassen ist lediglich von $\S 13$ SGB V durchbrochen. Nach §13 Abs. 3 Satz 1 SGB $\vee$ hat die Krankenkasse Kosten in der entstandenen Höhe nur zu erstatten, soweit die Leistung notwendig war, weil die Krankenkasse eine unaufschiebbare Leistung entweder nicht rechtzeitig erbringen konnte oder sie eine Leistung zu Unrecht 
abgelehnt hat und dadurch dem Versicherten für die selbstbeschaffte Leistung Kosten entstanden sind.

Ein derartiger Ausnahmefall vom Sachleistungsprinzip lag in dem vorliegenden Fall vor. Zu den Leistungen zählen neben Therapiemethoden ebenso Methoden zur Diagnostik, wie hier die streitgegenständliche PET-CT/MRT Untersuchung. Hierbei handelt es sich nicht um eine Standardtherapie, sondern um eine neue Untersuchungs- und Behandlungsmethode.

Die PET-CT erweitert die Leistungsfähigkeit der Diagnostik in der Onkologie. Die PET erkennt das Krebsgewebe aufgrund eines gesteigerten Stoffwechsels der Tumorzellen, die CT zeichnet eine genaue anatomische 3D-Landkarte des Körpers. Durch die Kombination beider bildgebenden Verfahren kann der Tumor präzise lokalisiert werden, sodass dem Patienten ein beschwerlicher „Diagnosemarathon" - und nicht selten auch Operationen auf reinen Verdacht hin - erspart werden können. Von der beantragten PETCT können zudem konkrete Aussagen zur Vitalität der Lymphknoten mit Metastasen erwartet werden. Diese Erkenntnisse sind erforderlich, um weitere Faktoren zur Einstellung in eine erneute Risiko/NutzenAbwägung hinsichtlich der Fortführung der Chemotherapie zu gewinnen.

Allerdings fehlt es für die bei dem Patienten vorliegende medizinische Indikation Hodentumor an einer positiven Bewertung oder Empfehlung des G-BA für die PET-CT/ MRT. In dem Fall ist es nach der Rechtsprechung des BVerfG unter drei Voraussetzungen möglich, die Kosten für eine noch nicht anerkannte, neue Untersuchungs- und Behandlungsmethode zu übernehmen:

1. Es muss eine lebensbedrohliche oder regelmäßig tödlich verlaufende Erkrankung vorliegen.

2. Für diese Erkrankung darf keine allgemein anerkannte, dem medizinischem Standard entsprechende Behandlung zur Verfügung stehen.

3. Durch die Behandlung muss eine nicht ganz fernliegende Aussicht auf Heilung oder wenigstens auf eine spürbar positive Einwirkung auf den Krankheitsverlauf bestehen.
Diese ausnahmsweisen Voraussetzungen sieht das erkennende Gericht als erfüllt an:

Zu 1): Unstreitig handelt es sich bei dem metastasierten Hodenkarzinom um eine potenziell lebensbedrohliche Erkrankung.

Zu 2): Eine andere als die vom Patienten in Anspruch genommene PET-CT/MRT Untersuchung stand zum damaligen Zeitpunkt nicht zur Verfügung. Diese Diagnosemethode hatte erhebliche Konsequenzen für das weitere Vorgehen zur Behandlung der Krankheit. Durch die PET-CT/MRT sollte festgestellt werden, ob eine toxische Chemotherapie - mit dem Risiko erneuter lebensbedrohlicher Nebenwirkungen - notwendig oder eine abwartende Haltung ausreichend war.

Zu 3): Die nach bundesverfassungsgerichtlicher Rechtsprechung nunmehr eingeführte Bestimmung des $§ 2$ Abs. 1a Satz 1 SGB V, welche eine spürbare positive Einwirkung auf den Krankheitsverlauf fordert, ist im Falle des klagenden Patienten ebenfalls erfüllt. Diese Bestimmung ist im Bereich der Untersuchungsmethoden dahingehend zu verstehen, dass die aus der Untersuchung gewonnenen Erkenntnisse von entscheidender Bedeutung für das weitere diagnostische und therapeutische Vorgehen sein müssen.

§2 Abs. 1a SGB V umfasst demzufolge nicht nur Ansprüche, die unmittelbar auf therapeutische Maßnahmen gerichtet sind (ebenso: Bundessozialgericht (BSG), Urteil vom 24.04.2018, Az.: B 1 KR 29/17 R); er bezieht sich vielmehr auch auf jede Leistung, die nicht entsprechend dem allgemein anerkannten medizinischen Standard zur Verfügung steht und die als Mittel der Erkenntnisgewinnung im Rahmen der Planung des weiteren therapeutischen Vorgehens hilft. Deshalb stellen auch Untersuchungen Leistungen der gesetzlichen Krankenversicherung dar, weil diese notwendige Voraussetzung zur Ermittlung der erforderlichen medizinischen Maßnahmen bei der Krankenbehandlung im Sinne des §27 SGB V sind. Mithin verlangt die Vorschrift des $\S 2$ Abs. 1a SGB V nur, dass durch die Leistung eine nicht ganz entfernt liegende Aussicht auf Heilung oder auf eine spürbar positive Einwirkung auf den Krankheitsverlauf besteht.
Demzufolge können auch Untersuchungsleistungen, die noch nicht dem Qualitätsgebot entsprechen, zum Behandlungserfolg beitragen, sofern es keine allgemein anerkannte, dem medizinischen Standard entsprechende Diagnostik gibt oder die diesem Standard entsprechenden diagnostischen Methoden ausgeschöpft sind, ohne jedoch hinreichende Erkenntnisse für das weitere therapeutische Vorgehen ergeben zu haben. Insoweit können auch nicht anerkannte diagnostische Maßnahmen in Betracht zu ziehen sein, sofern im Falle einer lebensbedrohlichen oder regelmäßig tödlichen Erkrankung - oder einer zumindest wertungsmäßig vergleichbaren $\mathrm{Er}$ krankung - durch deren Anwendung erst der Weg für therapeutische Maßnahmen eröffnet werden kann, mit denen eine nicht ganz entfernt liegende Aussicht auf Heilung oder spürbare positive Einwirkung auf den Krankheitsverlauf verbunden ist. Die spürbare positive Einwirkung auf den Krankheitsverlauf kann somit auch darin liegen, lebensbedrohliche Risiken von Therapieoptionen überhaupt erst abzuklären (BSG, wie vor).

\section{Einordnung der Entscheidung}

Das BSG hatte mit dem in der Entscheidung des SG Leipzig zitierten Urteil vom 24.04.2018, Az.: B 1 KR 29/17 R hinsichtlich PET-CT Untersuchungen zuvor bereits grundsätzlich klargestellt, dass Versicherte unter den Voraussetzungen grundrechtsorientierter Leistungsauslegung auch noch nicht allgemein anerkannte Untersuchungsmethoden einschließlich diagnostischer Maßnahmen beanspruchen können, um Therapieentscheidungen vorzubereiten und dass eine spürbare positive Einwirkung auf den Krankheitsverlauf auch darin liegen kann, die lebensbedrohlichen Risiken von Therapieoptionen abzuklären. Hierbei hatte das BSG jedoch keine verbindliche Entscheidung im Einzelfall treffen können, sondern hierzu an das Landessozialgericht (LSG) zur erneuten Verhandlung und Entscheidung zurückverwiesen. Die Entscheidung des SG Leipzig steht damit in einer Linie mit dem durch das BSG vorgezeichneten Weg und überträgt die durch das BSG aufgestellten Grundsätze auf die eigene Entscheidung im konkreten Fall. 


\section{Keine CT-Abrechnungs- genehmigung für zugelassene Nuklearmedizinerin}

Mit Beschluss vom 17.03.2021, Az.: B 6 KA 27/20 B hat das BSG eine Entscheidung des Bayerischen LSG bestätigt, nach der einer Fachärztin für Nuklearmedizin die Genehmigung für Leistungen der Computertomographie (CT) versagt ist.

\section{Sachverhalt}

Eine Fachärztin für Nuklearmedizin mit Zulassung zur vertragsärztlichen Versorgung und zugleich Fachärztin für Diagnostische Radiologie beantragte die Genehmigung zur Ausführung und Abrechnung von CT-Leistungen nach der Vereinbarung von Qualifikationsvoraussetzungen gemäß $\S 135$ Abs. 2 SGB V zur Durchführung von Untersuchungen in der diagnostischen Radiologie und Nuklearmedizin und von Strahlentherapie, der Antrag wurde durch die zuständige Kassenärztliche Vereinigung (KV) zurückgewiesen. Das LSG hat die Ablehnung der KV mit der Begründung bestätigt, die Ärztin erfülle als Fachärztin für Diagnostische Radiologie mit nachgewiesenen eingehenden Kenntnissen, Erfahrungen und Fertigkeiten in der CT-Diagnostik zwar die in der Vereinbarung normierten fachlichen Voraussetzungen für die Erteilung der Genehmigung. Jedoch könne sie als allein zugelassene Ärztin für Nuklearmedizin CT-Leistungen nicht fachgebietskonform erbringen. Wenn - wie im Fall der in einem Methodenfach (Nuklearmedizin) zugelassenen Ärztin, die keine Leistung eines anderen Methodenfachs (Radiologie) erbringen könne - von vornherein ausgeschlossen sei, dass von der Genehmigung im Rahmen der vertragsärztlichen Versorgung Gebrauch gemacht werden könne, seien Fachkundegenehmigungen nicht zu erteilen.
Mit ihrer Beschwerde wendet sich die Ärztin gegen die Nichtzulassung der Revision durch das LSG.

\section{Begründung}

In der Rechtsprechung des erkennenden Senats des BSG wird zwischen dem Fachkundenachweis auf der Grundlage von Vereinbarungen nach $\S 135$ Abs. 2 SGB $\vee$ und der Frage unterschieden, welche genehmigungsbedürftigen Leistungen ein Arzt unter Beachtung der Grenzen seines Fachgebietes erbringen darf. Lediglich die - auch hier einschlägige - Frage, ob ein für ein Methodenfach zugelassener Arzt unter dem Aspekt der Fachfremdheit eine Fachkundegenehmigung für die Leistungen eines anderen Methodenfaches erhalten kann, hat der Senat negativ beantwortet. Fachkundebezogene Genehmigungen muss die $\mathrm{KV}$ in solchen Fällen nicht erteilen, weil ausgeschlossen ist, dass der Arzt davon im Rahmen der vertragsärztlichen Versorgung Gebrauch machen kann.

Der Ärztin ist jedoch einzuräumen, dass der Senat für die fehlende Abrechenbarkeit fachfremder Leistungen auf die in den Heilberufs- und Kammergesetzen der Länder und in den von den Ärztekammern der Länder erlassenen Weiterbildungsordnungen (WBO) normierte Verpflichtung der Ärzte, die eine Gebietsbezeichnung führen, ihre Tätigkeit auf dieses Fachgebiet zu beschränken, abstellt. Berufsrechtlich ist die Ärztin berechtigt, beide Fachgebietsbezeichnungen (Fachärztin für Nuklearmedizin und Fachärztin für Diagnostische Radiologie) zu führen. Jedoch steht diese Aussage des Senats nicht für sich allein, sondern es wird sogleich ein Bezug zu der Tätigkeit des Arztes als Vertragsarzt hergestellt. So betont der Senat stets, dass Beschränkungen des Fachgebiets den Arzt auch in seiner Tätigkeit als Vertragsarzt erfassen. Selbst ein Arzt, der be- rufsrechtlich mehrere Gebietsbezeichnungen führen darf, aber nur für ein Fachgebiet zur vertragsärztlichen Versorgung zugelassen ist, muss sich im Rahmen der vertragsärztlichen Versorgung auf Leistungen des Gebietes beschränken, für das er zugelassen ist. Dementsprechend kommt es auch hier für die Frage der Fachfremdheit nur auf das Fachgebiet der Nuklearmedizin an, für das allein die Ärztin über einen Versorgungsauftrag verfügt.

\section{Einordnung der Entscheidung}

Die Entscheidung mag für die betroffene Doppelfachärztin ungünstig sein. Gleichwohl sind die Argumentation und das Ergebnis des BSG nachvollziehbar. Die Zulassung zur vertragsärztlichen Versorgung für ein Fachgebiet folgt aus der Bedarfsplanung. Hiernach ist die Erbringung fachfremder Leistungen grundsätzlich nicht vorgesehen und daher auch nicht möglich. Ist die Erbringung und Abrechnung der Leistungen nicht möglich, besteht seitens der KV auch keine Grundlage, um diesbezüglich Genehmigungen zu erteilen.

\section{Prof. Dr. Peter Wigge \\ Rechtsanwalt \\ Fachanwalt für Medizinrecht}

Tilmann Kirsch

Rechtsanwalt

Fachanwalt für Medizinrecht

Rechtsanwälte Wigge

Großer Burstah 42

20457 Hamburg

Telefon: (040) 3398 705-90

Telefax: (040) 3398 705-99

Internet: www.ra-wigge.de

E-Mail: kanzlei@ra-wigge.de 